\title{
GENERAL CASE OF MOVEMENT OF SOLID SYSTEM WITH SLIDER-CRANK MECHANISMS AS INTERNAL MOVERS ON ROUGH SURFACE WITH FRICTION
}

\author{
SERGEY V. SEMENDYAEV \\ Moscow Institute of Physics and Technology (MIPT) \\ 9 Institutskiy per., Dolgoprudny, Moscow Region, 141701, Russian Federation \\ semendyaevsergey@gmail.com, https://mipt.ru/english/
}

Key words: Solid System, Internal Mover, Slider-Crank Mechanism, Friction, Mathematical Modelling.

\begin{abstract}
The equations of motion in the mathematical model of the solid system with slidercrank mechanisms as internal movers are obtained in the general case of translational-rotational motion on a rough plane with dry friction. Viscous friction with linear dissipation is also considered in the model.
\end{abstract}

\section{INTRODUCTION}

Previously, solid state systems at three support points, driven by internal movers, were described [1-3].

There are several articles [4-8] on the movement with three points of support, but we have an important difference here and in articles [1-3] - the presence of internal movers in the system.

At the same time, there are articles from other researchers on internal movers, but there is no movement at the three points of support [9-12].

Earlier [1], for the considered mechanical system, equations of motion on a surface with friction were obtained in two cases: translational motion, rotational motion. In this paper, we consider the general case, including simultaneous translational and rotational motion.

It is worth mentioning that in other papers, the author also considers systems moving due to internal movers - systems with two massive eccentrics [2-3]. In general, interest in such systems is due to an unusual way of movement - without using standard movers, such as wheels, legs, tracks.

In this article the equations of motion in the mathematical model of the solid system with slider-crank mechanisms as internal movers are obtained in the general case of translationalrotational motion on a rough plane with dry friction. Viscous friction with linear dissipation is also considered in the model.

\section{MECHANICAL SYSTEM AND GENERAL STATEMENTS}

The mechanical system is presented in Fig. 1. It has a carrier frame on three points of support. Two slider-crank mechanisms are attached to the frame, which move the sliders relative to the frame. There is no friction between the frame and the sliders. Friction between the support 
points and the horizontal rough surface (let us call it earth) is either dry, described by the law of Coulomb, or viscous, depending linearly on speed.

The center of the mass of the frame and two points of sliders form a triangle of masses.

Three points of support on which the frame stands on the ground form a supporting triangle.

The movement of the system is due to the anisotropic pulsation of the mass triangle inside the supporting triangle. The movement of the support triangle by inertia is extinguished by friction. Thus, the movement occurs by repetitive pushes of sliders.

The frame is always in contact with the ground via support points.

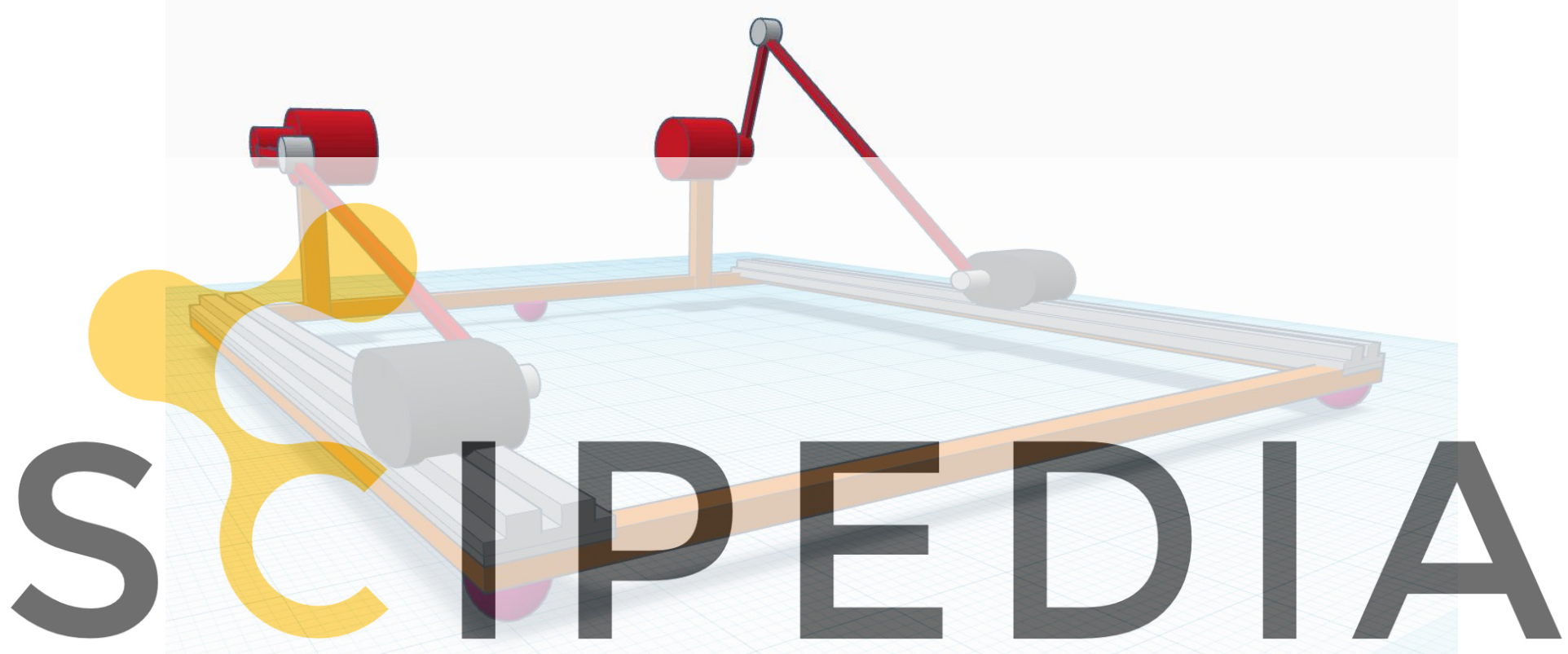

The movement of sliders is controlled by motors installed at the base of the cranks. Let us assume that the torques of motors allow to realize the control of the rotation angles of cranks, as the functions of time.

\section{MATHEMATICAL MODEL}

Let us identify the points at which the masses are concentrated (see Fig. 2) as follows:

$$
S_{i}, i=1,2,3
$$

where $S_{1}$ - center of mass of the carrier frame, $S_{2}, S_{3}$ - positions of masses of sliders (considered as points), sliding along the side ribs of the carrier frame, due to slider-crank mechanisms. 


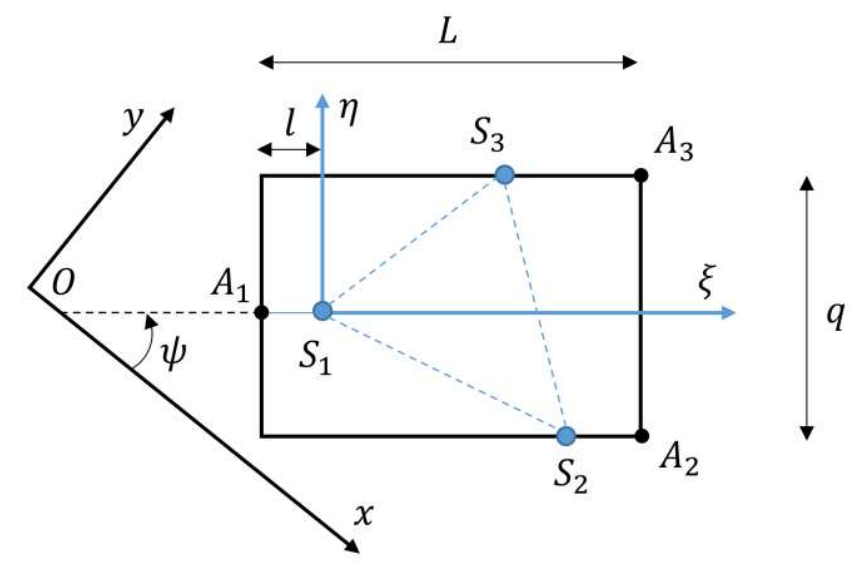

Figure 2: Geometric model of the mechanical system (view from above)

The distribution of the mass of the mechanical system will be defined as follows: the carrier frame has mass $m$ and a moment of inertia $J$, masses of sliders are accepted as point and equal to $\mu$ :

$$
m\left(S_{1}\right)=m, \quad m\left(S_{2}\right)=m\left(S_{3}\right)=\mu .
$$

So, we have a triangle of masses $S_{1} S_{2} S_{3}$, that changes its geometry.

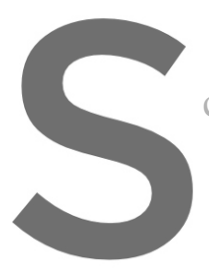

As we have already called ground.

The points of contact of the
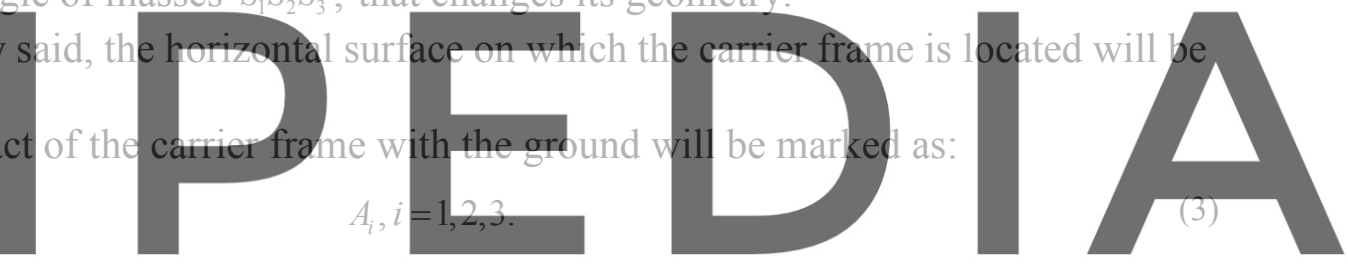

So we have a supporting triangle $A_{1} A_{2} A_{3}$, which has a constant geometry.

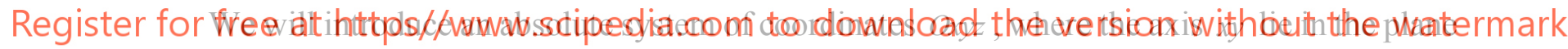

of the ground.

We will also introduce a relative system of coordinates $S_{1} \xi \eta \zeta$, associated with the canrier frame, where the axis $\xi \eta$ lie in the plane above the ground.

We will designate the vector of the position in the system $O x y z$ with a letter $r$, and in the system $S_{1} \xi \eta \zeta$ - with a letter $\rho$. We will also use the following brackets to indicate a vector component in a reference system: $\{0,0,0\}=(\circ, \circ, \circ)^{T}$.

Then for the points of the mass triangle in the $S_{1} \xi \eta \zeta$ we have:

$$
\begin{aligned}
& \rho\left(S_{1}\right)=\{0,0,0\}, \\
& \rho\left(S_{2}\right)=\left\{\xi_{2},-\frac{q}{2}, 0\right\}, \\
& \rho\left(S_{3}\right)=\left\{\xi_{3}, q / 2,0\right\},
\end{aligned}
$$

where $q=$ const - the transverse size of the carrier frame, and $\xi_{2}=\xi_{2}(t), \xi_{3}=\xi_{3}(t)$ take as time functions that control the system. In our case, they depend on the movement of slider-crank 
mechanisms through functions [1]:

$$
\xi_{i}(t)=a \sin \varphi_{i}(t)+\left(b^{2}-\left(h+a \cos \varphi_{i}(t)\right)^{2}\right)^{1 / 2}, \quad i=2,3 .
$$

where the geometric parameters of the slider-crank mechanism (for both sliders they have the same design) are shown on the Fig. 3.

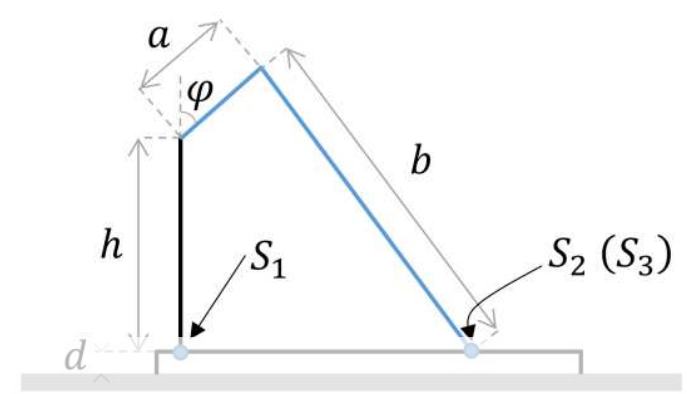

Figure 3: Geometric parameters of the slider-crank mechanism (view from the side)

The relative positions of sliders when moving are obviously in a limited range of values:

$$
\xi_{i} \in\left[\xi_{\min }, \xi_{\max }\right], \quad i=2,3 .
$$

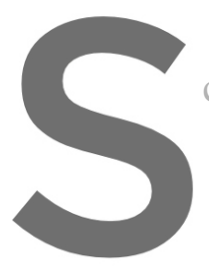

\section{For points of the} coordinates:
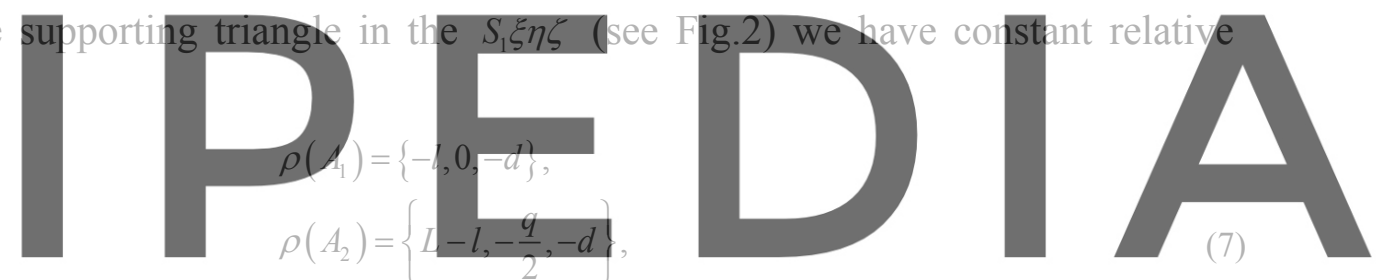

Register for free at https//www.scipedia.com to downhload the version without the watermark

where $L=$ const - longitudinal size of the carrier frame, $l=$ const - longitudinal displacement of the point of support $A_{1}$ relative to the center of mass $S_{1}$ of the frame, $d=$ const - height of the frame above the ground.

Now let us write down the coordinates of the points in the system Oxyz .

For a key point $S_{1}$, which is on the one hand the coordinate of the center of the mass of the carrier frame, and on the other hand the beginning of the relative system of coordinates, we have:

$$
r\left(S_{1}\right)=\{x, y, d\} .
$$

Then, for any point $P$ from the set $S_{i}, A_{i}, i=1,2,3$, we can write in the absolute coordinate system $O x y z$ :

$$
r(P)=r\left(S_{1}\right)+A_{\psi} \cdot \rho(P),
$$

where $A_{\psi}$ - rotation matrix from $S_{1} \xi \eta \zeta$ to $O x y z$ : 


$$
A_{\psi}=\left(\begin{array}{ccc}
\cos \psi & -\sin \psi & 0 \\
\sin \psi & \cos \psi & 0 \\
0 & 0 & 1
\end{array}\right),
$$

where $\psi$ - this is the angle of the carrier frame in the absolute coordinate system.

So, from (9) we have:

$$
\begin{aligned}
& r\left(S_{2}\right)=\left\{\frac{1}{2} q S_{\psi}+x+C_{\psi} \xi_{2},-\frac{1}{2} q C_{\psi}+y+S_{\psi} \xi_{2}, d\right\}, \\
& r\left(S_{3}\right)=\left\{-\frac{1}{2} q S_{\psi}+x+C_{\psi} \xi_{3}, \frac{1}{2} q C_{\psi}+y+S_{\psi} \xi_{3}\right\}, \\
& r\left(A_{1}\right)=\left\{-l C_{\psi}+x,-l S_{\psi}+y, 0\right\}, \\
& r\left(A_{2}\right)=\left\{(L-l) C_{\psi}+\frac{1}{2} q S_{\psi}+x,-\frac{1}{2} q C_{\psi}+(L-l) S_{\psi}+y, 0\right\}, \\
& r\left(A_{3}\right)=\left\{(L-l) C_{\psi}-\frac{1}{2} q S_{\psi}+x, \frac{1}{2} q C_{\psi}+(L-l) S_{\psi}+y, 0\right\},
\end{aligned}
$$

where we denoted $C_{\psi}=\cos \psi, S_{\psi}=\sin \psi$.

Let us write down cinematic values for key elements of the system.

The angular speed of the frame is defined as:
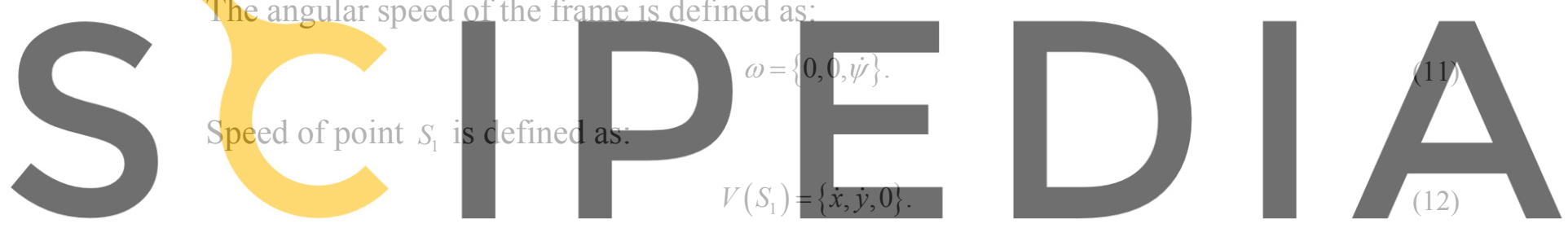

For points of the supporting triangle, we have in projections on the axis Oxyz:

Register for free at https//wwW.scipedia.com to download the version without the watermark $V\left(A_{i}\right)=V\left(S_{1}\right)+A_{\psi} \cdot\left(\omega \times \rho\left(A_{i}\right)\right) \cdot$

Hence, for the support points we have:

$$
\begin{aligned}
& V\left(A_{1}\right)=\left\{\dot{x}+l S_{\psi} \dot{\psi}, \dot{y}-l C_{\psi} \dot{\psi}, 0\right\}, \\
& V\left(A_{2}\right)=\left\{\dot{x}+\frac{1}{2} q C_{\psi} \dot{\psi}-(L-l) S_{\psi} \dot{\psi}, \dot{y}+\frac{1}{2} q S_{\psi} \dot{\psi}+(L-l) C_{\psi} \dot{\psi}, 0\right\}, \\
& V\left(A_{3}\right)=\left\{\dot{x}-\frac{1}{2} q C_{\psi} \dot{\psi}-(L-l) S_{\psi} \dot{\psi}, \dot{y}-\frac{1}{2} q S_{\psi} \dot{\psi}+(L-l) C_{\psi} \dot{\psi}, 0\right\} .
\end{aligned}
$$

For the points of the mass triangle in the Oxyz:

$$
V\left(S_{i}\right)=V\left(S_{1}\right)+A_{\psi} \cdot\left(\omega \times \rho\left(S_{i}\right)\right)+A_{\psi} \cdot \dot{\rho}\left(S_{i}\right),
$$

where the first two components are speed due to the movement of the frame in the projection on the axis $O x y z$, the third is the speed relative to the frame in the projection on the axis $O x y z$.

Hence, for the sliders we have: 


$$
\begin{aligned}
& V\left(S_{2}\right)=\left\{\dot{x}+\frac{1}{2} q C_{\psi} \dot{\psi}-\xi_{2} S_{\psi} \dot{\psi}+C_{\psi} \dot{\xi}, \dot{y}+\frac{1}{2} q S_{\psi} \dot{\psi}+\xi_{2} C_{\psi} \dot{\psi}+S_{\psi} \dot{\xi}_{2}, 0\right\}, \\
& V\left(S_{3}\right)=\left\{\dot{x}-\frac{1}{2} q C_{\psi} \dot{\psi}-\xi_{3} S_{\psi} \dot{\psi}+C_{\psi} \dot{\xi}, \dot{y}-\frac{1}{2} q S_{\psi} \dot{\psi}+\xi_{3} C_{\psi} \dot{\psi}+S_{\psi} \dot{\xi}_{3}, 0\right\} .
\end{aligned}
$$

Let us write down Newton's law of motion for the system:

$$
m \cdot \dot{V}\left(S_{1}\right)+\mu \cdot \sum_{i=2}^{3} \dot{V}\left(S_{i}\right)=\sum_{i=1}^{3} N_{i}+\sum_{i=1}^{3} F_{i}+(M+2 \mu) \cdot G
$$

where $G=\{0,0,-g\}$ - acceleration of free fall, $N_{i}=\left\{0,0, n_{i}\right\}$ - normal reaction at the point of support $A_{i}, F_{i}-$ Coulomb friction force, acting at the point $A_{i}$ :

$$
\begin{aligned}
& F_{i}=-k \cdot n_{i} \cdot V\left(A_{i}\right) \cdot\left(\left|V\left(A_{i}\right)\right|\right)^{-1}, \quad V\left(A_{i}\right) \neq 0, \\
& \left|F_{i}\right| \leq k \cdot n_{i}, \quad V\left(A_{i}\right)=0,
\end{aligned}
$$

where $k$-dry-friction coefficient.

In the case of $V\left(A_{i}\right)=0$ we are dealing with the static friction force. In this case, the force of friction depends on the dynamic state of the system, not on the speed $V\left(A_{i}\right)$.

Or, if we have viscous friction (linear dissipation), then we have:
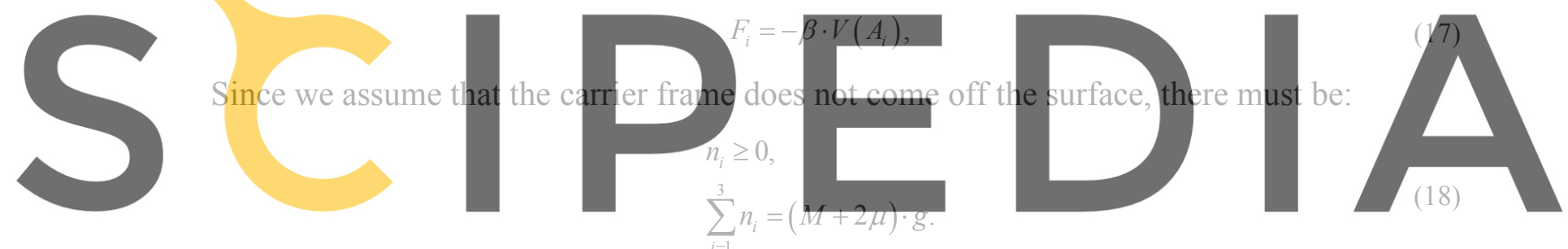

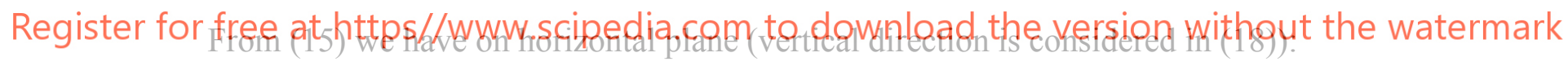

$$
(m+2 \mu) \cdot\left(\begin{array}{l}
\ddot{x} \\
\ddot{y}
\end{array}\right)+\mu \cdot\left(\begin{array}{l}
\left(C_{\omega}-S_{\psi} \omega\right)\left(\dot{\xi}_{2}+\dot{\xi}_{3}\right)-\left(\xi_{2}+\xi_{3}\right)\left(S_{\omega} \omega+S_{\psi} \varepsilon\right)+C_{\psi}\left(\ddot{\xi}_{2}+\ddot{\xi}_{3}\right) \\
\left(S_{\omega}+C_{\psi} \omega\right)\left(\dot{\xi}_{2}+\dot{\xi}_{3}\right)+\left(\xi_{2}+\xi_{3}\right)\left(C_{\omega} \omega+C_{\psi} \varepsilon\right)+S_{\psi}\left(\ddot{\xi}_{2}+\ddot{\xi}_{3}\right)
\end{array}\right)=\left(\begin{array}{l}
\sum_{i=1}^{3} F_{i x} \\
\sum_{i=1}^{3} F_{i j}
\end{array}\right)
$$

where $\omega=\dot{\psi}, \varepsilon=\ddot{\psi}, C_{\omega}=\cos \dot{\psi}, S_{\omega}=\sin \dot{\psi}$.

Let us write down the angular momentum of the system:

$$
K_{O}=m \cdot r\left(S_{1}\right) \times V\left(S_{1}\right)+J \cdot \Omega+\mu \cdot \sum_{i=2}^{3} r\left(S_{i}\right) \times V\left(S_{i}\right),
$$

where $\Omega=\{0,0, \omega\}$, and $J$ is the moment of inertia of the carrier frame for the axis $S_{1} \zeta$. Then, from (19) we have:

$$
\begin{gathered}
K_{O x}=-\dot{y} m d-\mu d\left(2 \dot{y}+C_{\psi}\left(\xi_{2}+\xi_{3}\right) \omega+S_{\psi}\left(\dot{\xi}_{2}+\dot{\xi}_{3}\right)\right), \\
K_{O y}=\dot{x} m d+\mu d\left(2 \dot{x}-S_{\psi}\left(\xi_{2}+\xi_{3}\right) \omega+C_{\psi}\left(\dot{\xi}_{2}+\dot{\xi}_{3}\right)\right),
\end{gathered}
$$




$$
\begin{aligned}
& K_{O z}=m(x \dot{y}-y \dot{x})+J \omega+ \\
& +\mu\left(-2 y \dot{x}-S_{\psi} \xi_{2} \dot{x}-S_{\psi} \xi_{3} \dot{x}+2 x \dot{y}+C_{\psi} \xi_{2} \dot{y}+C_{\psi} \xi_{3} \dot{y}+\frac{1}{2} q^{2} C_{\psi}^{2} \omega\right)+ \\
& +\mu\left(\frac{1}{2} q^{2} S_{\psi}^{2} \omega+C_{\psi} x \xi_{2} \omega+S_{\psi} y \xi_{2} \omega+C_{\psi}^{2} \xi_{2}^{2} \omega+S_{\psi}^{2} \xi_{2}^{2} \omega\right)+ \\
& +\mu\left(C_{\psi} x \xi_{3} \omega+S_{\psi} y \xi_{3} \omega+C_{\psi}^{2} \xi_{3}^{2} \omega+S_{\psi}^{2} \xi_{3}^{2} \omega+\frac{1}{2} q C_{\psi}^{2} \dot{\xi}_{2}\right)+ \\
& +\mu\left(\frac{1}{2} q S_{\psi}^{2} \dot{\xi}_{2}+S_{\psi} x \dot{\xi}_{2}-C_{\psi} y \dot{\xi}_{2}-\frac{1}{2} q C_{\psi}^{2} \dot{\xi}_{3}-\frac{1}{2} q S_{\psi}^{2} \dot{\xi}_{3}+S_{\psi} x \dot{\xi}_{3}-C_{\psi} y \dot{\xi}_{3}\right)
\end{aligned}
$$

For the rate of change of the angular momentum we have:

$$
\frac{d K_{O}}{d t}=\sum_{i=1}^{3} r\left(A_{i}\right) \times\left(F_{i}+N_{i}\right)+m \cdot r\left(S_{1}\right) \times G+\mu \cdot \sum_{i=2}^{3} r\left(S_{i}\right) \times G
$$

But it is not possible to write down briefly the result of the calculation of the expression (20) within this article.

According to obtained equations, we can consider the following possible tasks.

A) All three legs slide. Subtasks: A1 - the frame rotates; the friction forces are not parallel; A2 - the frame is moving translationally, and this means that all the friction forces are parallel.

B) Support point

$A_{1}$ we have the force

C) Support point

$A_{2}$ we have the force

D) Support point
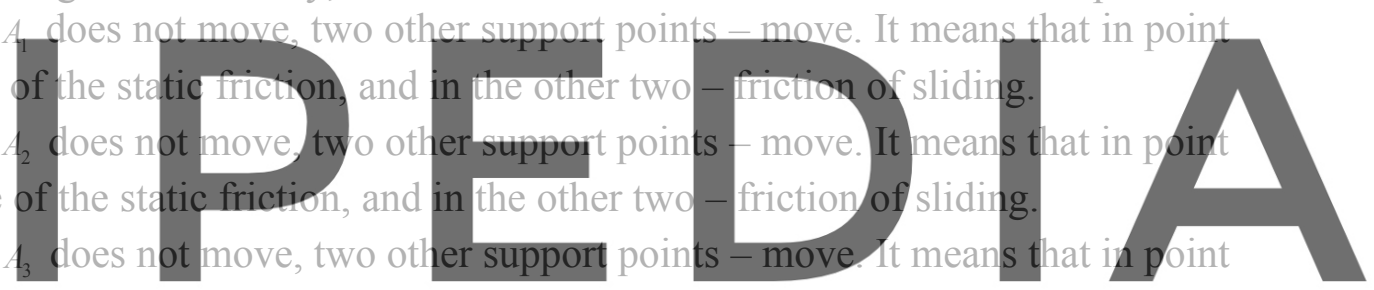

$A_{3}$ we have the force of the static friction, and in the other two - friction of sliding.

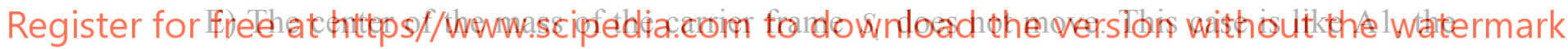
only difference is that out of all the variety of rotations we highlight one in which $V\left(S_{1}\right)=0$.

\section{CONCLUSIONS}

- $\quad$ Equations for general case of motion of the solid system with slider-crank mechanisms as internal movers are obtained on rough surface with friction.

- $\quad$ In the future, calculations are planned for systems with internal movers, considering the combined or multi-component dry friction model [13-17].

\section{ACKNOWLEDGEMENTS}

This work is partially supported by the Russian Foundation for Basic Research, project № 1801-00335.

\section{REFERENCES}

[1] Semendyaev, S.V. Coupled dynamics of solid system with slider-crank mechanisms as internal movers on rough surface with friction. Proceedings of the VII International 
Conference on Coupled Problems in Science and Engineering (COUPLED PROBLEMS 2017) (2017) 185-196 (ISBN: 978-84-943928-3).

[2] Semendyaev, S.V. Solid system with two massive eccentrics on a rough plane: rotational case. IFAC-PapersOnLine (2018) 51(2):884-889.

[3] Semendyaev, S.V. General case of movement of solid system with two massive eccentrics on a rough plane. Proceedings of the VIII International Conference on Coupled Problems in Science and Engineering (COUPLED PROBLEMS 2019) (2019) 183-191 (ISBN: 97884-949194-5-9).

[4] Field, P. On the motion of a disc with three supports on a rough plane. Phys. Rev. (Series I). (1912) 35:177.

[5] Shegelski, M.R.A. and Goodvin, G.L. and Booth, R. and Bagnall, P. and Reid, M. Exact normal forces and trajectories for a rotating tripod sliding on a smooth surface. Canad. $J$. of Physics. (2004) 82(11):875-890.

[6] Rosenblat, G.M. On the integration of the equation of motion of the body, based on a rough plane by three points. Dokl. Akad. Nauk (2010) 435(4):475-478. (in Russian)

[7] Borisov, A.V. and Mamaev, I.S. and Erdakova, N.N. Dynamics of a body sliding on a rough plane and supported at three points. Theor. and Appl. Mechanics. (2016) 43(2):169-190.

[8] Rozenblat, G.M. Motion of a body sliding on a rough horizontal plane and supported at three points. Doklady Physics (2017) 62(9):430-433.

[9] Chernous'ko, F.L. and Bolotnik, N.N. and Figurina, T.Yu. Optimal control of vibrationally excited locomotion systems. Reg and Chaotic Dynam. (2013) 18(1-2):85-99.

[10] Ivanov, A.P. and Sh rotor, on a rough plat

11] Volkova, L.Yu. environment. Nelin

[12] Sakharov, A.V. Rotation o mass on a rough plane. Reg. and Chaotic Dynam. (2015) 20(4):428-440.

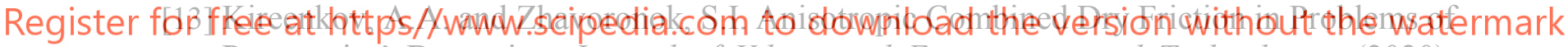
Pneumatics' Dynamics, Journal of Vibrational Engineering and Technologies (2020), 8 (2): 365-372 (DOI: 10.1007/s42417-019-00140-1).

[14] Kireenkov, A.A. Improved friction model of the aviation tyre contact with the landing strip. IFAC-PapersOnLine (2018) 51(2): 890-894 (DOI 10.1016/j.ifacol.2018.04.027)

[15] Kireenkov, A.A., Zhavoronok, S.I. Implementation of analytical models of the anisotropic combined dry friction in problems of pneumatics' dynamics. MATEC Web of Conferences (2018) 211(08004) (DOI 10.1051/matecconf/201821108004)

[16] Kireenkov, A.A., Nushtaev, D.V., Zhavoronok, S.I. A new approximate model of tyre accounting for both deformed state and dry friction forces in the contact spot on the background of the coupled model. MATEC Web of Conferences (2018) 211(08003) (DOI 10.1051/matecconf/201821108003)

[17] Kireenkov, A.A., Zhavoronok, S.I., Nushtaev, D.V. On tire models accounting for both deformed state and coupled dry friction in a contact spot. Computer Research and Modeling (2021) 13(1):163-173 (DOI: 10.20537/2076-7633-2021-13-1-163-173) 